\title{
UWB MST MEMS-Based Near-Field Imaging System
}

\author{
S. Capdevila ${ }^{1}$, M. Jofre ${ }^{1}$, J. Rodriguez ${ }^{2}$, M. Guardiola ${ }^{1}$, A. Papió ${ }^{1,2}$, \\ F. De Flaviis ${ }^{2}$, and L. Jofre ${ }^{1}$ \\ ${ }^{1}$ AntennaLab, Universitat Politècnica de Catalunya (UPC), Barcelona, \\ Spain, e-mail: scapdevila@tsc.upc.edu \\ ${ }^{2}$ University of California, Irvine, USA
}

\section{Introduction}

The capability of microwave signals to penetrate and sense into materials with reasonable spatial resolution makes them attractive for different industrial, medical and security applications where optical imaging systems are not applicable [1],[2]. Wideband signals such as those produced by ultra wide band (UWB) systems [3], and in particular the lower band of the recent 3.1-10.6 GHz band, offer new possibilities to increase spatial resolution and material electrical parameter measurement accuracy [4].

Near-field imaging systems are usually based on the measurement of the diffracted field produced by the object under test when illuminated by a controlled incident field. In order to reduce perturbation, acquisition time and RF complexity, modulated scattering technique (MST) has been proved to be a good solution for field measurement in general and for imaging systems [5] in particular. Existing systems are in general tuned or tuneable over a certain frequency band while for UWB an instantaneous wide frequency band behaviour is needed.

In this paper a new family of illuminators and MST measurement architectures is presented and the different aspects are studied.

\section{The near field imaging system}

The system represented in Fig. 1 consists in a UWB illuminator able to create a uniform incident field over the exploration area, and a UWB measurement array created by a UWB MST-probe array combined by a collecting antenna. A rectangular exploration area with $L_{X}=20 \mathrm{~cm}$ and $L_{Y}=20 \mathrm{~cm}$ is defined. For both the illuminator and collector antennas a conformed Vivaldi [6] configuration has been chosen with dimensions $L_{V}=23 \mathrm{~cm}, L_{A}=20 \mathrm{~cm}$ whose profiles have been optimized to produce a quasi uniform amplitude and phase distribution over its output aperture, over the 3.1 to $10 \mathrm{GHz}$ bandwidth. Fig. 2, presents the comparison of the simulated and measured electrical field distribution at $1 \mathrm{~cm}$ of the antenna aperture at 3 and $10 \mathrm{GHz}$.

In order to measure a significant amount of the fields scattered by the object under test, we should be able to collect the diffracted field over a set of points $p_{i}$ (e.g. linear or circular line, for the 2D case); a real N-probe or a synthetic N-position array needs to be created over those points and connected through an RF circuit to the receiver, this is one of the elements that will increase the complexity of the 


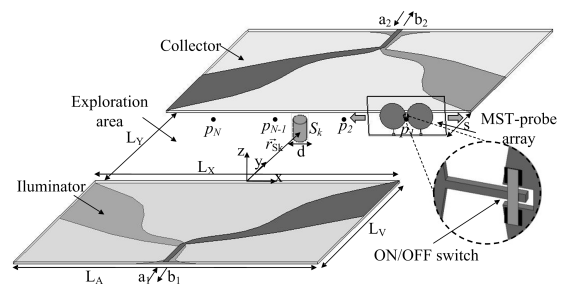

Figure 1: Measurement set-up for MST MEMS-based imaging system

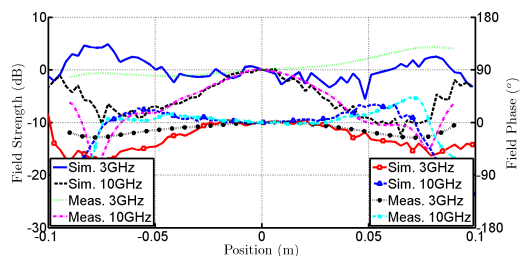

Figure 2: Near Field at $1 \mathrm{~cm}$ of the edge of the Vivaldi antenna

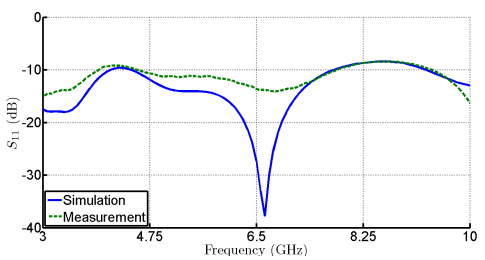

Figure 3: Comparison of the $S_{11}$ of the probe antenna

system as well as contribute to a perturbation effect in the measurement. The MST technique avoids the use of the $\mathrm{RF}$ connection and allows to reduce the acquisition time through an electronic addressing scheme. The whole system is characterized as a two-port microwave system, taking $E_{p_{i}}^{(1)}$ and $E_{p_{i}}^{(2)}$ as the electrical field at the probe position $p_{i}$, created by the illuminator and collector antennas when fed by an incident signal $a_{1}$ and $a_{2}$ respectively, and by loading the probe antenna with a modulated load $Z_{L}$, the MST technique, allows us to relate the field at the probe position with the field reradiated and received at the illuminator antenna input $b_{1}^{m}$ (reflection technique) or at the collector antenna output $b_{2}^{m}$ (transmission technique) as:

$$
S_{11}=\frac{b_{1}^{m}}{a_{1}} \propto\left(E_{p_{i}}^{(1)}\right)^{2} \quad S_{21}=\frac{b_{2}^{m}}{a_{1}} \propto\left(E_{p_{i}}^{(1)} E_{p_{i}}^{(2)}\right)
$$

The transmission technique obtains a better dynamic range (as long as the field $E_{p_{i}}^{(2)}$ at the aperture of the collector antenna can be considered almost uniform) while requires of a second antenna. In this case:

$$
S_{21}=\frac{b_{2}^{m}}{a_{1}} \propto\left(E_{p_{i}}^{(1)}\right)
$$

In order to obtain the UWB character, a wide band antenna and modulating devices are needed. For the probe antenna a dipole disc antenna [7] has been used, Fig. 3, presents a comparison of the simulated and measured $S_{11}$ covering the $3.1-10 \mathrm{GHz}$ band. For the modulating element, MEMS device due to its wideband response, constitutes an appropriate switching element. Fig. 4, represents the ON-OFF load states into the frequency band.

\section{The experimental near-field imaging system results}

To do an initial validation of the imaging capabilities of the system, experimental UWB measurements (3.1-10.6 GHz) have been done. Measurements were taken 

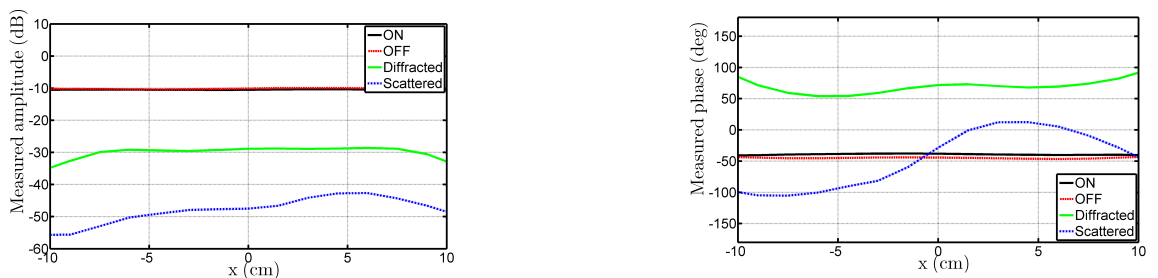

Figure 4: $S_{21}$ for the ON, OFF measurements and the differential and scattered field estimations at $3 \mathrm{GHz}$.

inside an anechoic chamber in order to avoid excessive reflections from the environment. A simple point-like metallic element (diameter $=1.6 \mathrm{~cm}$ ) was placed inside the exploration area $\left(20 x 20 \mathrm{~cm}^{2}\right)$ at $\mathrm{x}=5 \mathrm{~cm}$ and $\mathrm{y}=16 \mathrm{~cm}$ and the measuring process consisted of a set of measurement where the disc antenna probe is moved along the $20 \mathrm{~cm}$ of the scanning line. A total of 15 measurements were done, moving the disc antenna along the edge of the collector antenna with a stepping of $1.5 \mathrm{~cm}$. An UWB extension of the Fourier Back-propagation method [8] has been used to relate the measured scattered field $E^{s}\left(x, z_{0}\right)$ with the image $I(x, y)$ as:

$$
I(x, y)=F T^{-1}\left\{\frac{2 j k_{y}}{k^{2}} \cdot F T\left\{E^{s}\left(x, y_{0}\right)\right\} e^{j k_{y}\left(y_{m}-y\right)}\right\}
$$

Fig. 5 represents the comparison of the simulated and measured results, and Fig. 6, shows the image obtained over the exploration area.
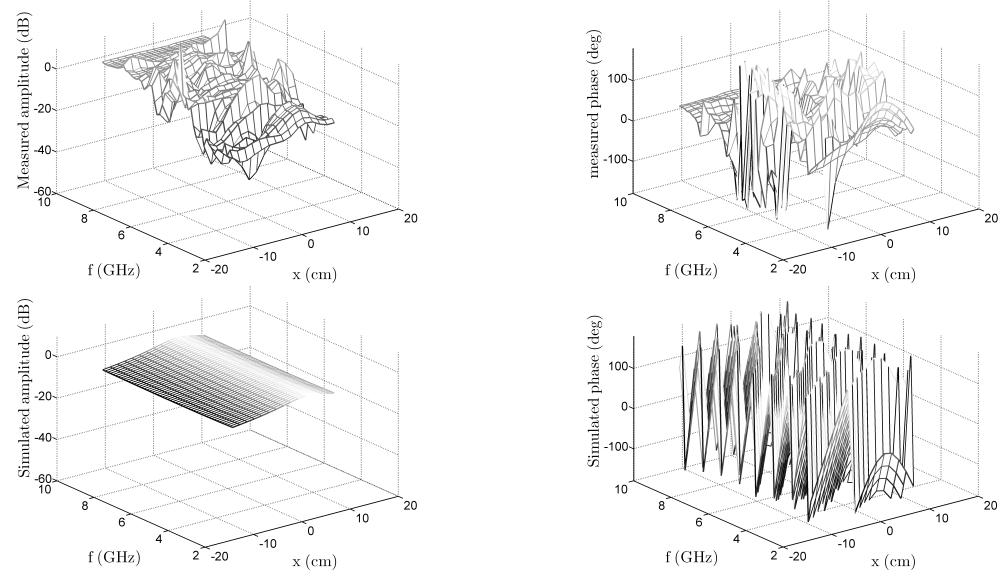

Figure 5: Comparison of the measured and simulated results for the scattered field at the disc-antenna along the scanning line from $3-10 \mathrm{GHz}$.

\section{Conclusions}

A complete system for UWB imaging has been discussed and characterized. The system uses conformed Vivaldi geometries for the illuminator and collector antennas, and a MEMS switched disc dipole as the probe.An imaging algorithm has been presented for a linear transmission imaging architecture. A complete experimental validation has been done for the Vivaldi antennas, probe antenna and switching 


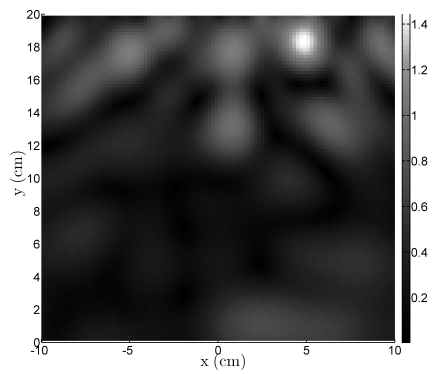

Figure 6: Reconstructed image obtained over the exploration area.

MST technique. Preliminary results have been obtained for a point-like object, showing good results in accordance with UWB resolution capabilities.

\section{Acknowledgments}

This work has been supported by the CICYT through grant TEC2007-66698-C0401/TCM, the Spanish Government through FPU program and the Balsells Generalitat de Catalunya-University of California, Irvine Engineering Innovation Program.

\section{References}

[1] R. Zoughi, Microwave Testing and Evaluation. Amsterdam, The Netherlands: Kluwer, 2000.

[2] J. C. Bolomey, Frontiers in Industrial Process Tomography. London, U.K.: Engineering Foundation, 1995.

[3] R. Zetik, J. Sachs, and R. S. Thoma, "UWB short-range radar sensing - the architecture of a baseband, pseudo-noise uwb radar sensor," Instrumentation $\mathscr{G}$ Measurement Magazine, IEEE, vol. 10, no. 2, pp. 39-45, April 2007.

[4] A. Papió, J. Jornet, P. Ceballos, J. Romeu, S. Blanch, A. Cardama, and L. Jofre, "UWB short range imaging," St. Louis, Missouri, USA, 27-29 June 2007.

[5] J. C. Bolomey and F. E. Gardiol, Engineering Applications of the Modulate Scattering Technique. Artech House, 2001.

[6] H. G. Schantz, "Introduction to ultra-wideband antennas," in Conference on Ultra Wideband Systems and Technologies, 16-19 Nov. 2003, pp. 1-9.

[7] Y. Taeyoung, S. Seong-Youp, R. Nealy, W. A. Davis, and W. L. Stutzman, "Compact antennas for uwb applications," in Conference on Ultra Wideband Systems and Technologies, 16-19 Nov. 2003, pp. 205-208.

[8] J. M. Rius, C. Pichot, L. Jofre, J. C. Bolomey, N. Joachimowicz, A. Broquetas, and M. Ferrando, "Planar and cylindrical active microwave temperature imaging: numerical simulations," Medical Imaging, IEEE Transactions on, vol. 11, no. 4, pp. 457-469, Dec 1992. 